\title{
Does Morphine Influence Blood Count in Palliative Patients? : A Longitudinal Study from an Oncology Center in Nepal
}

\section{Sirisa Karki ${ }^{1^{*}}$, Sameer Timilsina ${ }^{2}$ and Sabitri Sharma ${ }^{3}$}

${ }^{1}$ Department of Pharmacology, Tribhuwan University, Chitwan Medical College, Bharatpur-5, Chitwan, Nepal

${ }^{2}$ Department of Physiology, Tribhuwan University, Chitwan Medical College, Bharatpur-5, Chitwan, Nepal

${ }^{3}$ Palliative Care Unit, Nepal Cancer Hospital and Research Center, Harisiddhi, Lalitpur, Nepal

*Corresponding Author: Sirisa Karki, Department of Pharmacology, Tribhuwan University, Chitwan Medical College, Bharatpur-5, Chitwan, Nepal, Tel: +977-9841346624; E-mail: sirisa.karki@gmail.com

Received date: May 03, 2018; Accepted date: May 29, 2018; Published date: June 04, 2018

Copyright: ( 2018 Karki S, et al. This is an open-access article distributed under the terms of the Creative Commons Attribution License, which permits unrestricted use, distribution, and reproduction in any medium, provided the original author and source are credited.

\begin{abstract}
:
Introduction: Morphine has been the cornerstone of pain management in palliative care patients. The easy accessibility and availability of opioids at most tertiary care centers of Nepal have made possible, the once thought impossible fundamental right of palliative patients. The availability of morphine has not only eased the pain of palliative patients but health care providers equally. The mainstay of pain management has had its success but several subjective complaints of dry mouth, sedation, feeling of incomplete wellbeing and decreased immune function is often heard of. The present study aims at evaluating the effects of morphine on complete blood count profile in palliative care patients.
\end{abstract}

Methods: Complete blood count of 114 palliative patients was estimated using an automatic hematology analyzer after 24-48 hours and 14 days of morphine administration. The data was recorded using a preformed proforma and analyzed using SPSS (20).

Results: A statistically significant increase in total white blood cell count, neutrophil and platelet count was observed following morphine administration but there was a decrease in lymphocyte count $(p<0.05)$. Also, neutrophil-lymphocyte ratio, a predictor of inflammation was found to increase $(p<0.05)$. A non-significant increase was seen with the platelet-lymphocyte ratio $(p>0.05)$.

Conclusion: Morphine is found to influence the total white blood cells, neutrophils, and lymphocytes and NLR in palliative patients. However, withholding morphine in palliative patients is never an option. Furthermore, larger randomized controlled trials are necessary to analyze the predictive value of NLR and PLR in palliative patients.

Keywords: Morphine; Opioids; Blood count; Neutrophil; Lymphocyte

Abbreviations: NLR: Neutrophil Lymphocyte Ratio; PLR: Platelet Lymphocyte Ratio; TC: Total Count; QoL: Quality of Life; WHO: World Health Organization

\section{Background}

Palliative care as defined by World Health Organization is "an approach that improves the quality of life (QoL) of patients and their families facing the problems associated with life-threatening illness, through the prevention and relief of suffering by means of early identification and impeccable assessment and treatment of pain and other problems, physical, psychosocial and spiritual" [1]. Though palliative services started in Nepal since the turn of the 21st century, it has not been able to reach a large population living in the remote parts of the country because of localized centers in urban areas [2]. Pain is one of the most distressing symptoms in palliative patients and when not treated effectively has a detrimental effect on QoL [3]. Opioids especially morphine has been the cornerstone of pain management in palliative patients. The regular and easy availability of morphine since
2008 in the country has not only eased the physical pain of palliative patients, the mental pain of practicing physicians has also decreased significantly [4]. The morphine consumption statistics has consistently been low and below the global standard as only $6 \%$ of the cancer patients have access to this fundamental right.

With a rise in the use of oral morphine for treating pain in palliative patients, a number of side-effects of the drugs arise. The side effects of dry mouth (95\%), sedation and constipation (88\%), myoclonus (83\%) and nausea (50\%) are often underestimated [5]. Apart from these side effects, morphine has been documented to strongly influence the immune function of the human body acting as an immunosuppressant [6-8]. It has been suggested that morphine affects both the innate and adaptive immunity of the host [9]. Morphine suppresses the phagocytic function of neutrophil by inhibiting the release of nitric oxide (NO) release mediated by $\mu-3$ receptor subtype present in the immunocytes [10]. Also, morphine acting on its opioid receptor depresses lymphocyte proliferation [11]. In spite of its immunosuppression activity, morphine continues to be used extensively in palliative patients for symptomatic relief of pain. Also, Neutrophil Lymphocyte Ratio (NLR) and Platelet Lymphocyte ratio (NLR) has recently gained attention in medical research as a marker of inflammation [12]. These simple, cost-effective yet reliable biomarkers 
are useful predictors of disease outcome [13]. In the present study, we aim to evaluate the white blood cell profile of palliative patients following initiation of morphine. Also, we aim to study the NLR and PLR in palliative patients following morphine treatment. No studies of such kind have been conducted in Nepal as of now and few studies surrounding the issue can be found in the literature.

\section{Methods}

This was a hospital-based longitudinal study conducted from July 2017 to December 2017 at Nepal Cancer Hospital and Research Center, Harisiddhi, Lalitpur, Nepal. A total of 120 palliative patients both admitted and attending the OPD were included in the study. Patients with advanced cancer on diagnosis, progressive disease, refractory disease, recurrent disease and those who did not warrant treatment either due to social or economic reasons were included in the study. Patients receiving active cancer treatment; chemotherapy or radiotherapy and those with hematological malignancies were not included in the study. A written consent was obtained from all the patients for participation in the study. A complete blood count of the 120 palliative patients was estimated using an automatic hematology analyzer 24-48 hours after initiation of morphine therapy. After 2 weeks of continued morphine therapy, complete blood count was again performed in 114 patients (6 patients passed away during the period) and recorded in a pre-tested porforma. Laboratory standard operation procedures were maintained for all laboratory analysis. Internal quality control sera, both normal and pathological, were also run for each lot, for the validation of the results. Statistical analyses were done by SPSS 20.0 version (Statistical Package for Social Science). Shapiro-Wilk test was done to find the normality of the distribution and paired sample "t-test" was done to compare the hematological values obtained on two occasions.

\section{Results}

A total of 120 patients [51.7\% (62) males and 48.3\% (58) females] were included in the study. Six of them passed away within 2 weeks of morphine and were excluded from the study. Among the remaining 114 patients, the mean age was $62.31 \pm 2.56$ years ranging from 36 years to 88 years. The median age was 68 years. The most common carcinoma was Non-small cell lung cancer (NSCLC) (42.6\%) followed by GI cancers (28.3\%), gynecological malignancies (19.3\%).

The normally distributed variables were total count (TC), neutrophils $(\mathrm{N})$, monocytes $(\mathrm{M})$, eosinophils $(\mathrm{E})$ and platelets $(\mathrm{P})$. The non-normally distributed variables included the lymphocytes $(\mathrm{L})$, the NLR and PLR. Table 1 shows the mean values of all the variables and its correlation.

The average neutrophils count increased from $72.37 \pm 9.71$ to $78.00 \pm 10.66$ whereas the average lymphocytes count was found to decrease from $23.95 \pm 10.36$ to $20.40 \pm 10.36$. The increase in mean neutrophils and a decrease in mean lymphocytes increased the neutrophil-lymphocyte ratio. The average total count was found to increase whereas the average platelet count was found to decrease.

\begin{tabular}{|l|l|l|l|}
\hline \multirow{2}{*}{ Parameters } & \multicolumn{2}{|c|}{ Mean } & \multirow{2}{*}{-value } \\
\cline { 2 - 4 } & 24 hours & $\mathbf{1 4}$ days & \\
\hline TC (/mm3) & $8367.46 \pm 2668.61$ & $10206.58 \pm 3363.17$ & $<0.05$ \\
\hline Neutrophils (\%) & $72.37 \pm 9.71$ & $78.00 \pm 10.66$ & $<0.05$ \\
\hline Lymphocytes (\%) & $23.95 \pm 10.36$ & $20.40 \pm 10.36$ & $<0.05$ \\
\hline Platelets (/mm3) & $254833.33 \pm 88671.90$ & $222964.91 \pm 66195.33$ & $<0.05$ \\
\hline NLR & $4.00 \pm 3.61$ & $5.41 \pm 4.47$ & $<0.05$ \\
\hline PLR & $13728.29 \pm 13201.73$ & $14441.23 \pm 11282.480$ & NS \\
\hline
\end{tabular}

Table 1: Mean values of hematologic parameters after 24 hours and 14 days of morphine administration along with their correlation.

\section{Discussion}

The male to female predominance was around 1.06 which is similar to other studies of the region. The predicted rise in female cases to male cases by 2020 could as well be true [14]. Also, the most common condition was carcinoma of the lung which has been reported as commonest cancer in Nepal [15].

We observed a net rise in mean white blood cell count and neutrophil count in palliative patients. The rise in both total white blood cell count and neutrophil count was statistically significant $(\mathrm{p}<0.05)$. We predicted that the rise in both total white blood cell count and neutrophil count was because of the morphine therapy. Here, the absolute neutrophil count also increased on day 14 and the increase was also statistically significant. The rise of white blood cell count and neutrophils could as well have been a result of increased risk of infection as a result of immuno-compromised status irrespective of morphine use. Some reports have suggested morphine to decrease white blood cell count. The present study was dissimilar with that very finding, rather reported an increase in white blood cell count and neutrophils count.

There was a decrease in mean lymphocyte on day 14 of morphine administration which was also statistically significant $(\mathrm{p}<0.05)$. Morphine suppresses peripheral blood lymphocyte proliferation via sympathetic nervous system stimulation $[11,16]$. The average lifespan of a lymphocyte is ranged from weeks to months and as the blood tests were performed in 2 weeks, the subtle decrease might not have been appreciated. Some studies in the past have attributed a decrease in lymphocyte number to apoptosis. But some failed to report a decrease even when human cells were studied for apoptosis $[17,18]$. The present study is in no condition to agree or disagree the molecular mechanism of the decrease but is only in a state to report a decrease in the average lymphocyte count after initiation of morphine therapy. 
The NLR was found to increase on the 14th day. NLR as of recent times has been used as a simple yet useful biomarker of inflammation [19]. The increase in the NLR has been found to inversely influence the overall survival. NLR has also been used as a marker to study cancer progression $[13,20]$. As the patients in the study were only under supportive care aiming to improve the QoL, the progression of disease was not under doubt. The increase in NLR could have some predictive value in palliative patients. NLR could be useful in estimating survival and preparing for the end of life care and decision [21].

We also observed an increase in the PLR but this was not statistically significant $(\mathrm{p}>0.05)$. Unlike NLR, PLR has not been used as an efficient marker in the latter stages of life but is equally invaluable as NLR in predicting the prognosis of the disease [22].

The acute effect of opioids on the endocrine system is believed to either reduce or not change the ACTH (Adreno-Corticotrophic Hormone) or glucocorticoids, however, with the chronic administration it leads to a decrease in HPA (Hypothalamo-PituitaryAdrenal) axis activity. The influence on this axis produces more glucocorticoids which act as an immuno-suppressor but the exact role of morphine in such cases is highly debatable and understudied. Also, it has been suggested that the presence of cancer and chronic diseases potentially alters the nutritional status of the person leading to immunosuppression. The release of immunosuppressive cytokines in terminally ill patients has also been advocated to influence the immune system.

In Nepal, where religious beliefs and social dogmas are regarded invaluable, several home remedies like wheat, maize and barley grass juice, ayurvedic medicines and medicinal herbs were given to ease the sufferings of patients. The role of those substances if any on blood profile was not considered. The socioeconomic status of the patients with reference to their nutritional status beforehand was also not analyzed.

Also, the patients in the study were all prescribed morphine in doses greater than $80 \mathrm{mg} /$ day to as high as $400 \mathrm{mg} /$ day. The dose-dependent relationship of morphine with blood profile was not studied.

\section{Conclusion}

An increased total white cell count, neutrophils, and platelets with decreased lymphocyte count can be anticipated following morphine administration in palliative patients. Inflammatory biomarkers i.e., NLR and PLR which are predictors of overall survival also increase after morphine therapy. The readily available and cost-effective inflammatory biomarker (NLR) warrants further investigation as to establish itself as a reliable prognostic tool.

Though it influences blood counts, morphine therapy should not be withheld in palliative patients as it has been found to improve QoL.

\section{Declarations}

\section{Ethics approval and consent to participate}

Ethical approval was obtained from Institutional Review Committee of Nepal Cancer Hospital and Research Center, Harisiddhi, Lalitpur, Nepal. A written consent was obtained from all the palliative patients for participation in the study. The consent informed the participants that the data obtained could be used and made public under anonymity. All procedures were in accordance with the Helsinki declaration and its later amendments.

\section{Consent to publish}

Not Applicable

\section{Availability of data and material}

The datasets obtained and/or analyzed during the current study are not publicly available due to confidentiality consent of the study but can be obtained from the corresponding author on reasonable request.

\section{Competing interests}

The authors declare that they have no competing interests.

\section{Funding}

No funding of any sort was received for the study.

\section{Authors' contribution}

SK conceptualized the study, wrote the initial proposal, participated in data collection and drafted the paper. ST assisted in drafting the initial manuscript, data entry and analysis and revising the draft. SS obtained the consent, collected the data, arranged follow-ups and collected the laboratory reports. All authors read and approved the final manuscript.

\section{Acknowledgments}

We would like to thank Dr. Sudip Shrestha, Chairman of Nepal Cancer Hospital and Research Center for providing us the opportunity to conduct the study under his supervision. We also would like to thank Dr. Bijesh Raj Ghimire, Medical Director and Consultant Oncologist for his valuable suggestions and feedback during the study. We would also like to thank Mrs. Bidhya Pathak Rai, Mr. Raj Kumar Gautam and Mr. Jeewan Dhimal at NCHRC. Also, thanks to the Institutional Review Committee of NCHRC for allowing us to conduct the study at this institute. Nonetheless, our sincere gratitude to all the participants in the study without whom it would have been impossible to conduct the study.

\section{References}

1. Definition of palliative care [http://www.who.int/cancer/palliative/ definition/en/]

2. Wright M, Wood J, Lynch T, Clark D (2008) Mapping levels of palliative care development: A global view. J Pain Symptom Manage 35: 469-485.

3. Katz N (2002) The impact of pain management on quality of life. J Pain Symptom Manage 24: S38-47.

4. Paudel BD, Ryan KM, Brown MS, Krakauer EL, Rajagopal MR, et al. (2015) Opioid availability and palliative care in Nepal: Influence of an international pain policy fellowship. J Pain Symptom Manage 49: 110-116.

5. Glare P, Walsh D, Sheehan D (2006) The adverse effects of morphine: A prospective survey of common symptoms during repeated dosing for chronic cancer pain. Am J Hosp Palliat Care 23: 229-235.

6. Boland JW, McWilliams K, Ahmedzai SH, Pockley AG (2014) Effects of opioids on immunologic parameters that are relevant to anti-tumour immune potential in patients with cancer: A systematic literature review. Br J Cancer 111: 866-873. 
Citation: Karki S, Timilsina S, Sharma S (2018) Does Morphine Influence Blood Count in Palliative Patients? : A Longitudinal Study from an Oncology Center in Nepal. J Pain Manage Med 4: 134.

Page 4 of 4

7. Hashiguchi S, Morisaki H, Kotake Y, Takeda J (2005) Effects of morphine and its metabolites on immune function in advanced cancer patients. J Clin Anesth 17: 575-580.

8. Al-Hashimi M, Scott SW, Thompson JP, Lambert DG (2013) Opioids and immune modulation: More questions than answers. Br J Anaesth 111 80-88.

9. Chang MC, Fan SZ, Hsiao PN, Cheng WF, Sun WZ (2011) Influence of morphine on host immunity. Acta Anaesthesiol Taiwan 49: 105-108.

10. Welters ID, Menzebach A, Goumon Y, Langefeld TW, Teschemacher H, et al. (2000) Morphine suppresses complement receptor expression, phagocytosis, and respiratory burst in neutrophils by a nitric oxide and $\mu_{3}$ opiate receptor-dependent mechanism. J Neuroimmunol 111: 139-145.

11. Bayer BM, Daussin S, Hernandez M, Irvin L (1990) Morphine inhibition of lymphocyte activity is mediated by an opioid dependent mechanism. Neuropharmacol 29: 369-374.

12. Cho KM, Park H, Oh D-Y, Kim TY, Lee K-H, et al. (2017) Usefulness of neutrophil-to-lymphocyte ratio, platelet-to-lymphocyte ratio, and their dynamic changes during chemotherapy to predict prognosis of advanced biliary tract cancer. J Clin Oncol 35: 416-416.

13. Zheng J, Cai J, Li H, Zeng K, He L, et al. (2017) Neutrophil to Lymphocyte Ratio and Platelet to Lymphocyte Ratio as Prognostic Predictors for Hepatocellular Carcinoma Patients with Various Treatments: A MetaAnalysis and Systematic Review. Cell Physiol Biochem 44 : 967-981.

14. Poudel KK, Huang Z, Neupane PR, Steel R (2017) Prediction of the Cancer Incidence in Nepal. Asian Pac J Cancer Prev 18: 165-168.
15. Piya MK, Acharya SC (2012) Oncology in Nepal South Asian J Cancer 1: 5-8.

16. Hall DM, Suo JL, Weber RJ (1998) Opioid mediated effects on the immune system: Sympathetic nervous system involvement. J Neuroimmunol 83: 29-35.

17. Ohara T, Itoh T, Takahashi M (2005) Immunosuppression by morphineinduced lymphocyte apoptosis: Is it a real issue? Anesth Analg 101: 1117-1122.

18. Sebastian Palm SL, Christian Gerd Leimenstoll, Christoph Mignat, Joerg Steinmann Maier (1998) Does Prolonged Oral Treatment with SustainedRelease Morphine Tablets Influence Immune Function? Anesth Analg 86: 166-172.

19. Hwang IC, Park Y, Lee YJ, Ahn HY (2017) Simple Biomarkers During the Last Month of Life of Terminal Cancer Patients: A Sequential Study. J Pain Symptom Manage 53: e1-e3.

20. Templeton AJ, McNamara MG, Seruga B, Vera-Badillo FE, Aneja P, et al. (2014) Prognostic role of neutrophil-to-lymphocyte ratio in solid tumors: A systematic review and meta-analysis. J Natl Cancer Inst 106: dju124.

21. Nakamura Y, Watanabe R, Katagiri M, Saida Y, Katada N, et al. (2016) Neutrophil/lymphocyte ratio has a prognostic value for patients with terminal cancer. World J Surg Oncol 16: 141-148.

22. Templeton AJ, Ace O, McNamara MG, Al-Mubarak M, Vera-Badillo FE, et al. (2014) Prognostic role of platelet to lymphocyte ratio in solid tumors: A systematic review and meta-analysis. Cancer Epidemiol Biomarkers Prev 23: 1204-1212. 